\title{
Ventricular Fibrillation Caused by Traumatic Coronary Artery Dissection
}

\author{
Jun-ya Ishikawa ${ }^{1}$, Naoto Morimura ${ }^{2 *}$, Eri Nagai ${ }^{3}$, Kyota Nakamura2 ${ }^{2}$ Makoto Shimizu ${ }^{4}$, \\ Keiji Uchida ${ }^{5}$ \\ ${ }^{1}$ Department of Emergency Medicine, Yokohama Municipal Citizen's Hospital, Yokohama, Japan \\ ${ }^{2}$ Advanced Critical Care and Emergency Medical Center, Yokohama City University Medical Center, Yokohama, \\ Japan \\ ${ }^{3}$ Department of Anesthesiology, Kanagawa Cancer Center, Yokohama, Japan \\ ${ }^{4}$ Department of Cardiology, International Goodwill Hospital, Yokohama, Japan \\ ${ }^{5}$ Cardiovascular Center, Yokohama City University Medical Center, Yokohama, Japan \\ Email: ${ }^{*}$ molimula@yokohama-cu.ac.jp
}

Received 12 March 2015; accepted 4 April 2015; published 8 April 2015

Copyright ( 2015 by authors and Scientific Research Publishing Inc.

This work is licensed under the Creative Commons Attribution International License (CC BY).

http://creativecommons.org/licenses/by/4.0/

c) (i) Open Access

\begin{abstract}
Coronary artery dissection due to blunt chest trauma (traumatic coronary artery dissection [TCAD]) may heal spontaneously, and some surgeons believe that conservative or elective treatments are sufficient, provided that there are no progressing ischemic symptoms. However, we report a patient who experienced sudden ventricular fibrillation (VF) during initial medical care for trauma injuries. The 32-year-old woman was riding in a passenger car when an accident occurred, and was subsequently transported to our emergency department. Twelve-lead electrocardiography revealed ST segment elevation in leads II, III, and aVF, although her vital signs remained stable. Therefore, we prioritized assessing the trauma at other sites, and VF suddenly occurred. Coronary angiography was performed with repeated defibrillation and chest compressions, which resulted in recovery of spontaneous circulation. Occlusion was observed in the right coronary artery, which we treated with balloon angioplasty. However, intravascular ultrasonography (IVUS) subsequently revealed coronary artery dissection, a stent was placed, and the patient successfully recovered. Therefore, IVUS may be useful for diagnosing TCAD, and swift intervention is needed if TCAD is detected.
\end{abstract}

\section{Keywords}

Coronary Artery Dissection, Blunt Chest Trauma, Ventricular Fibrillation, Acute Myocardial Infarction, Intravascular Ultrasonography

\footnotetext{
*Corresponding author.
}

How to cite this paper: Ishikawa, J., Morimura, N., Nagai, E., Nakamura, K., Shimizu, M. and Uchida, K. (2015) Ventricular Fibrillation Caused by Traumatic Coronary Artery Dissection. Case Reports in Clinical Medicine, 4, 119-123. 


\section{Introduction}

Traumatic coronary artery dissection (TCAD) is a life-threatening condition that can result in acute myocardial infarction (AMI). However, there are several reports of spontaneous healing, and some surgeons believe that conservative treatment is sufficient. In this report, we describe a patient who developed sudden ventricular fibrillation (VF) during our initial examination and treatment of blunt chest trauma.

\section{Case Report}

A previously healthy 32-year-old woman was involved in a traffic accident while she was riding in the passenger seat of a sedan. During the accident, she was pushed against the guardrail, experienced blunt chest trauma, and was subsequently transferred to our emergency department within 30 min of the accident. She smoked 10 15 cigarettes per day and had a family history of myocardial infarction (father), although there were no other risk factors for ischemic heart disease. At our examination, her Glasgow Coma Scale score was 14 (E3/V5/M6), her pulse was regular at 116/min, and her blood pressure was 130/104 $\mathrm{mmHg}$. The patient had a laceration on her left forehead, and complained of pain extending from the left neck region to the left shoulder and upper left side of her back. We observed hypesthesia and motor paralysis of her left arm, which was later diagnosed as a left brachial plexus injury.

Chest radiography revealed fracture of the left acromion, dislocation of the left first rib, and tracheal deviation to the right, although there was no evidence of pulmonary congestion. Twelve-lead electrocardiography (ECG) revealed sinus tachycardia, with ST segment elevation in leads II, III, and aVF, with reciprocal ST segment depression in leads I, aVL, and V2-V6 (Figure 1). In addition, we observed hypokinesia of the inferior left ventricle wall via transthoracic ultrasonography. A hematoma on the left side of her neck became apparent, and as the patient developed inspiratory stridor (likely induced by the hematoma), we performed tracheal intubation. Furthermore, as the patient's circulatory status was relatively stable, computed tomography (CT) was performed at the beginning of the secondary survey.

Immediately after the brain CT, which revealed no evidence of intracranial injury, the patient suddenly developed VF. Although recovery of spontaneous circulation was achieved via defibrillation and chest compressions, recurrent episodes of VF were observed, which required coronary angiography (CAG) at $3 \mathrm{~h}$ after the accident. We observed that the most proximal portion of her right coronary artery (RCA) appeared to be dilated
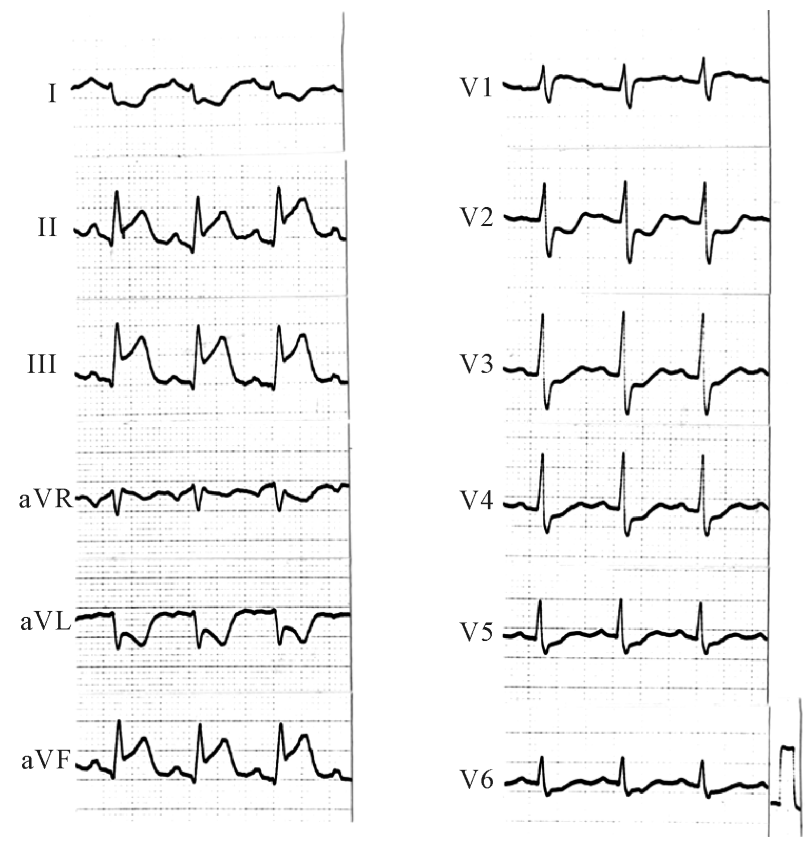

Figure 1. Electrocardiogram on arrival in emergency department showing ST segment elevation in leads II, III, aVF and reciprocal ST segment depression in I, aVL and V2-V6. 
and aneurysmal, with complete occlusion at the distal end (Figure 2(a)). However, the left coronary artery exhibited no evidence of either stenosis or atheromatous changes. Therefore, balloon angioplasty was performed, and complete patency of the RCA was restored at $4 \mathrm{~h}$ after the accident (Figure 2(b)). As subsequent examination via intravascular ultrasonography (IVUS) revealed dissection of the RCA (Figure 3(a)), we also performed stent placement, and subsequent IVUS no longer revealed a false lumen (Figure 3(b)). As the patient was at a high risk of hemorrhage from the other injury sites, we did not administer anticoagulant therapy.

The patient's subsequent recovery was uneventful; she was extubated on day 8 and was transferred from the intensive care unit to the general ward on day 9 without any complaints of chest pain or dyspnea. A follow-up CAG on day 19 confirmed the absence of any signs of restenosis, and the patient was discharged without any neurological deficits on day 22.

\section{Discussion}

Unfortunately, TCAD is a life-threatening disease that can cause AMI or lead to sudden VF in some cases. Therefore, it is important that TCAD be recognized early, and that early intervention be administered. In these cases, IVUS is useful for determining that occlusion of the coronary artery is not simple thrombosis, but rather that it is caused by dissection.

Chest pain or ECG abnormalities are often used to identify TCAD, and there are several reports where the TCAD resulted in VF during the initial examination and treatment [1]-[4]. However, TCAD can occasionally be

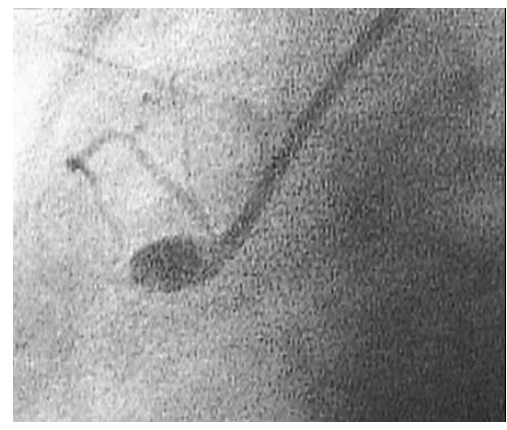

(a)

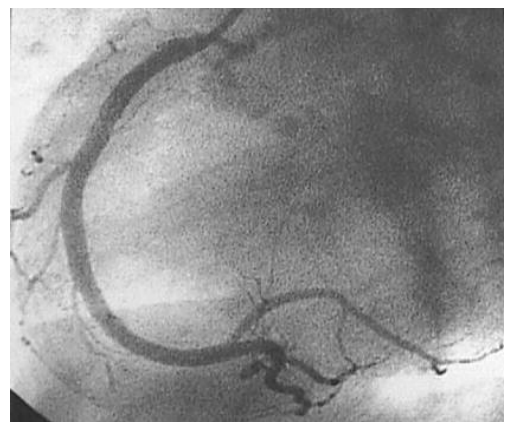

(b)

Figure 2. Coronary angiogram of right coronary artery (RCA): (a) Before balloon angioplasty. The proximal portion of the RCA dilated like aneurysm, and completely occluded at the distal end; (b) After balloon angioplasty. Complete recanalization of the RCA was obtained but coronary artery dissection findings were not visualized.

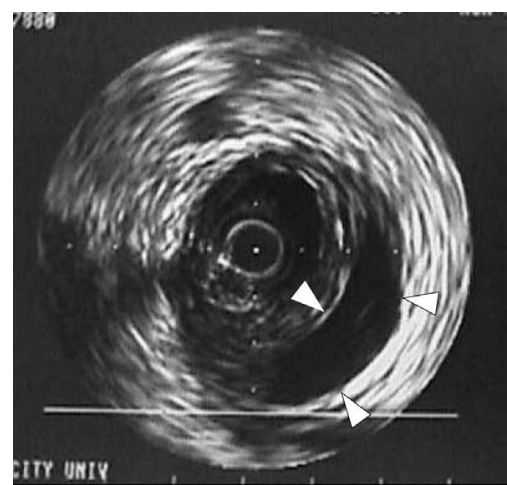

(a)

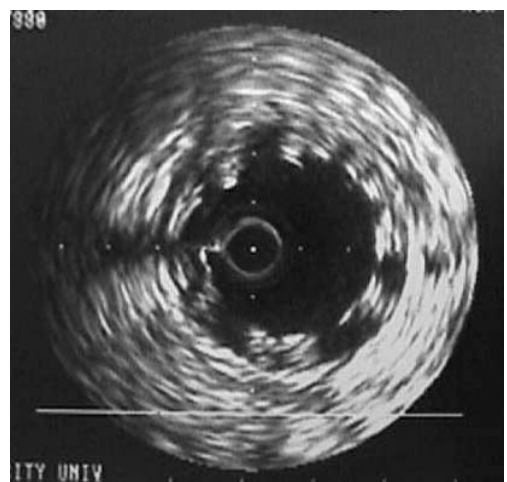

(b)

Figure 3. Intravascular ultrasonogram of right coronary artery: (a) Before placement of stent. Dissection was visualized as lunate low echoic space seen in the right lower part of this figure $(\Delta)$; (b) After placement of stent. The lunate low echoic space disappeared. 
resolved via conservative treatment, or may spontaneously heal during follow-up observation, and it has been argued that aggressive early intervention is unnecessary unless there is ongoing myocardial ischemia, involvement of proximal left coronary artery, or involvement of a large area of the myocardium [5]-[7]. Unfortunately, once TCAD develops into VF, the likelihood of survival is very low [8].

Similarly, the early mortality rate for non-traumatic AMI is high, and the direct cause of death is often VF or pulseless ventricular tachycardia (VT) [9]. In these cases, early reperfusion therapy is thought to reduce the occurrence of VF or pulseless VT, and subsequently improve the survival rate [10]. The pathology of myocardial ischemia due to sudden occlusion of the coronary artery is the same, regardless of the causal injury, and early reperfusion therapy is considered very important, even in cases of AMI that was caused by TCAD [2] [11]. In addition, TCAD often occurs proximal to the left coronary artery [12], which exposes a large section of the myocardium to ischemia. Furthermore, when TCAD causes sudden occlusion of the coronary artery in young people (who have not experienced ischemia), no collateral pathways exist in their hearts, which have not experienced ischemic preconditioning [13]. Therefore, in cases of TCAD in young patients, the immediate administration of reperfusion becomes even more critical. Although some cases have been alleviated via conservative therapy, there are also reported cases that followed a severe course, with outcomes that included heart failure, left ventricular aneurysm, systemic embolism from a left ventricular thrombus, or mechanical complications [14] [15]. These cases appear to indicate that myocardial necrosis is progressing as long as the coronary artery is occluded, even if spontaneous resolution is eventually observed. Therefore, early reperfusion is critical to preventing development into fatal arrhythmia, and can help to protect the myocardium, which can prevent severe pump failure or mechanical complications [8] [11] [16] [17].

In the present case, IVUS was useful for diagnosing the TCAD (which facilitated the placement of a stent), as the occluded RCA precluded identification of the dissection via CAG. Among 77 cases of AMI that were caused by blunt chest trauma, one study has reported that CAG indicated occlusion in $56.6 \%$ of the cases, and only $15.8 \%$ of the cases were found to have dissection [12]. However, the shear force that is applied to the coronary artery wall via rapid deceleration can cause intimal tearing, whereas the generation of thrombosis has typically been considered the pathogenesis of occlusion [6] [17]-[19]. Therefore, in cases where CAG indicates simple occlusion, it is very possible that undetected intimal tearing may have occurred [18] [19]. When such intimal tearing exists, balloon angioplasty alone may allow a thrombus to re-form at that site [16]. Moreover, dissection may lead to expansion of the false lumen, which would preclude thrombolysis [4] [7] [17]. Therefore, it is important to differentiate between dissection and simple occlusion in cases of chest trauma, and IVUS appears to be useful for this differentiation.

Coronary artery injury should be suspected if there is chest pain that cannot be explained by musculoskeletal injury or any observed ischemic change in the ECG findings that might indicate specific involvement of the coronary artery region. However, initial examination and treatment of blunt trauma may also require the assessment and treatment of various other injuries. Therefore, the assessment of coronary artery injury may be postponed in cases with relatively stable vital signs. However, as in the present case, it is possible for sudden (and potentially fatal) arrhythmia to be caused by coronary artery injuries, including coronary artery dissection. Thus, we believe that assessment and resuscitation (reperfusion) should be performed during the primary survey if there is inexplicable chest pain or ischemic change in the ECG, as described above.

\section{Conclusion}

TCAD is a life-threatening disease that can cause AMI or lead to sudden VF in some cases. Therefore, early recognition and intervention is critical. It is also important to differentiate between simple thrombosis/occlusion and dissection of the coronary artery, and we believe that IVUS is useful for this differentiation.

\section{Consent}

Written informed consent was obtained from the patient for publication of this case report and any accompanying images.

\section{Competing Interests}

The authors declare that they have no competing interests. 


\section{References}

[1] Greenberg, J., Salinger, M., Weschler, F., Edelman, B. and Williams, R. (1998) Circumflex Coronary Artery Dissection Following Waterskiing. Chest, 113, 1138-1140. http://dx.doi.org/10.1378/chest.113.4.1138

[2] Li, C.H., Chiu, T.F. and Chen, J.C. (2007) Extensive Anterolateral Myocardial Infarction Caused by Left Main Coronary Artery Dissection after Blunt Chest Trauma: A Case Report. The American Journal of Emergency Medicine, 25, 858.e3-e5.

[3] Dobias, M., Hejna, P. and Mawiri, A.A. (2013) Coronary Artery Dissection: A Fatal Complication Following Blunt Chest Trauma. Forensic Science, Medicine, and Pathology, 9, 454-457. http://dx.doi.org/10.1007/s12024-013-9442-6

[4] Kaplon-Cieslicka, A., Kosior, D.A., Grabowski, M., Rdzanek, A., Huczek, Z. and Opolski, G. (2013) Coronary Artery Dissection, Traumatic Liver and Spleen Injury after Cardiopulmonary Resuscitation-A Case Report and Review of the Literature. Archives of Medical Science, 9, 1158-1161. http://dx.doi.org/10.5114/aoms.2013.39235

[5] Grady, A.E., Cowley, M.J. and Vetrovec, G.W. (1985) Traumatic Dissecting Coronary Arterial Aneurysm with Subsequent Complete Healing. The American Journal of Cardiology, 55, 1424-1425. http://dx.doi.org/10.1016/0002-9149(85)90518-1

[6] Marik, P.E. (1990) Coronary Artery Dissection after a Rugby Injury. A Case Report. South African Medical Journal, 77, 586-587.

[7] Kerwin, T.C., Ruggie, N. and Klein, L.W. (2002) Spontaneous Coronary Artery Dissection Following Low-Intensity Blunt Chest Trauma: A Case Report and Review of Current Treatment Options. The Journal of Invasive Cardiology, 14, 679-681.

[8] Diaz-Miron, J.L., Dillon, P.A., Saini, A., Balzer, D.T., Singh, J., et al. (2014) Left Main Coronary Artery Dissection in Pediatric Sport-Related Chest Trauma. The Journal of Emergency Medicine, 47, 150-154. http://dx.doi.org/10.1016/j.jemermed.2014.04.034

[9] O’Connor, R.E., Brady, W., Brooks, S.C., Diercks, D., Egan, J., et al. (2010) Part 10: Acute Coronary Syndromes: 2010 American Heart Association Guidelines for Cardiopulmonary Resuscitation and Emergency Cardiovascular Care. Circulation, 122, S787-S817. http://dx.doi.org/10.1161/CIRCULATIONAHA.110.971028

[10] Antman, E.M., Anbe, D.T., Armstrong, P.W., Bates, E.R., Green, L.A., et al. (2004) ACC/AHA Guidelines for the Management of Patients with ST-elevation Myocardial Infarction: A Report of the American College of Cardiology/ American Heart Association Task Force on Practice Guidelines (Committee to Revise the 1999 Guidelines for the Management of Patients with Acute Myocardial Infarction). Circulation, 110, 588-636. http://dx.doi.org/10.1161/01.CIR.0000134791.68010.FA

[11] Bjornstad, J.L., Pillgram-Larsen, J. and Tonnessen, T. (2009) Coronary Artery Dissection and Acute Myocardial Infarction Following Blunt Chest Trauma. World Journal of Emergency Surgery, 4, 14. http://dx.doi.org/10.1186/1749-7922-4-14

[12] Christensen, M.D., Nielsen, P.E. and Sleight, P. (2006) Prior Blunt Chest Trauma May Be a Cause of Single Vessel Coronary Disease; Hypothesis and Review. International Journal of Cardiology, 108, 1-5. http://dx.doi.org/10.1016/j.ijcard.2005.04.010

[13] Morin, J., Bogaty, P.M., Roy, L. and Poirier, P. (2003) Trauma-Induced Occlusion of the Left Anterior Descending Artery Following a Snowmobile Accident in a Man without Prior Coronary Artery Disease. The Canadian Journal of Cardiology, 19, 565-568.

[14] Harada, H., Honma, Y., Hachiro, Y., Mawatari, T. and Abe, T. (2002) Traumatic Coronary Artery Dissection. The Annals of Thoracic Surgery, 74, 236-237. http://dx.doi.org/10.1016/S0003-4975(01)03515-9

[15] Goulah, R.D., Rose, M.R., Strober, M. and Haft, J.I. (1988) Coronary Dissection Following Chest Trauma with Systemic Emboli. Chest, 93, 887-888. http://dx.doi.org/10.1378/chest.93.4.887

[16] Fu, Z.L. and Yao, H.C. (2013) Percutaneous Coronary Intervention for Acute Myocardial Infarction Following Blunt Chest Trauma. International Journal of Cardiology, 168, 2983-2984. http://dx.doi.org/10.1016/j.ijcard.2013.04.100

[17] Ozdogan, O., Karacelik, M., Ekmekci, C. and Ozbek, C. (2013) Management of Acute Myocardial Infarction after a Blunt Chest Trauma. Turkish Journal of Trauma \& Emergency Surgery, 19, 173-176. http://dx.doi.org/10.5505/tjtes.2013.29291

[18] Shamsi, F., Tai, J.M. and Bokhari, S. (2014) Coronary Artery Dissection after Blunt Chest Trauma. BMJ Case Reports, Sep. 22. http://dx.doi.org/10.1136/bcr-2013-203520

[19] Li, X., Lei, Y. and Zheng, Q. (2014) Myocardial Infarction Caused by Coronary Artery Dissection due to Blunt Injury: Is Thromboaspiration an Appropriate Treatment? Hellenic Journal of Cardiology, 55, 61-64. 